\title{
Effects of Catchment Activities on Macrofaunal Assemblages in Tasmanian Estuaries
}

\author{
G. J. Edgar ${ }^{a, c}$ and N. S. Barrett ${ }^{b}$ \\ ${ }^{a}$ School of Zoology, University of Tasmania, GPO Box 252-05, Hobart, Tasmania, 7001 Australia \\ ${ }^{b}$ Tasmanian Aquaculture and Fisheries Institute, University of Tasmania, Crayfish Point Laboratory, Taroona, \\ Tasmania, 7053 Australia
}

Received 23 August 1999 and accepted in revised form 12 fanuary 2000

\begin{abstract}
Silt loadings associated with human activities in catchments were inferred to have an extremely widespread effect on estuarine macrobenthos around Tasmania. Estuaries with human population densities exceeding $10 \mathrm{~km}^{-2}$ in catchments consistently possessed muddy rather than sandy beds and shores, and were dominated by infauna rather than epifauna. Estuaries with human population densities below $1 \mathrm{~km}^{-2}$ in catchments possessed sandy sediments and numerous epifaunal species. These effects were consistent within groups of estuaries possessing similar hydrology and geomorphology. Although faunal composition differed substantially between estuaries possessing low and high human population densities, the number of macrofaunal species was similar. Population effects therefore could neither be detected using species richness indices, nor by the ABC method. Faunal changes were most clearly detected using disturbance indices weighted by the sensitivity of individual species to human activity. Two such indices, which are based on abundance $\left(D I_{n}\right)$ and productivity $\left(D I_{p}\right)$ data, are suggested to provide useful local indicators of estuarine health.
\end{abstract}

(C) 2000 Academic Press

Keywords: benthos; catchment processes; estuaries; human impacts; macrofauna; siltation; stress; Tasmania

\section{Introduction}

Shipping access, availability of dependable fresh water, prevalence of fertile alluvial land, and productivity of waters for fish and shellfish have led worldwide to the concentration of human activity on estuaries. Changes to estuarine ecosystems inevitably follow development as a consequence of factors such as port construction (Whitfield, 1986), channel dredging (van Dolah et al., 1984), introduction of exotic taxa (Carlton, 1989), damming of migration routes (Rosenberg et al., 1995), reduction in freshwater flows (Schlacher \& Wooldridge, 1996), siltation (Newcombe \& Jensen, 1996), marine farm pollution (Ritz et al., 1989; DeFur \& Rader, 1995), and by the direct removal of ecologically-important fish and invertebrate species. Estuaries also provide access to hinterland regions, and serve as conduits for sewage (Cloern, 1996) and fertiliser (Lavery et al., 1991), mine runoff (Winemillter \& Morales, 1989), industrial waste (Saiz-Salinas et al., 1996) and heated water (Bamber \& Spencer, 1984). The outcome of these activities is often eutrophication (Nixon, 1995; McClelland et al., 1997; Valiela et al., 1997) and

${ }^{c}$ Tel: +61 36226 7632; Fax: +61 36226 2745; E-mail: g.edgar@utas.edu.au macrophyte loss (Silberstein et al., 1986; Boynton et al., 1996), with nearly all estuaries outside polar regions suffering substantial anthropogenic degradation.

While the effects of human activity on estuarine ecosystems have been studied on numerous occasions, most studies have been confined to individual estuaries. More general assessments of the relative intensity of different human impacts at regional, national or international scales have not often been attempted (but see De Jonge et al., 1994). Hence in North America, for example, the question of whether the introduction of exotic species has caused greater disruption to estuarine communities than vegetation clearance remains unanswered. Clearly, the answer in this case will vary greatly between estuaries; however, information on net effects at regional scales is crucial when assessing research priorities. Funding on noticeable human impacts within a few badlydegraded urbanized estuaries may well have occurred at the expense of identifying more subtle impacts affecting estuarine processes throughout large regions.

The aim of the present study was to identify impacts on macrofaunal communities of human activity that extended over a regional scale of $\approx 1000 \mathrm{~km}$ around Tasmania. 


\section{Methods}

Samples of macrofauna retained on a $1 \mathrm{~mm}$ sieve were collected using $150 \mathrm{~mm}$ diameter corers inserted to a depth of $100 \mathrm{~mm}$ at 55 sites in 48 estuaries distributed around Tasmania and associated Bass Strait islands, as described in more detail in Edgar et al. (2000). Sites were selected haphazardly from the universe of estuarine sites around the coast and encompassed a wide range of environmental conditions, with salinities varying from 0 to 53 , tidal range varying from 0 to $2.3 \mathrm{~m}$, and estuary catchment area varying from 6 to $13100 \mathrm{~km}^{2}$. At each site, cores were collected using a spatiallynested sampling design based on (i) three random transect lines perpendicular to the shore $\approx 100 \mathrm{~m}$ apart, (ii) three to five fixed tidal heights down each transect (high water mark, mid tide level, low water mark, $0.3 \mathrm{~m}$ below low water, $0.7 \mathrm{~m}$ below low water), and (iii) two random replicates $\approx 1 \mathrm{~m}$ apart at each height down each transect.

In the laboratory, samples were sorted into sizeclasses using a nested series of sieves. The biomass of each animal $>2 \mathrm{~mm}$ sieve size was directly determined or estimated from length data and length/weight regressions. The biomass of animals $<2 \mathrm{~mm}$ sieve size was estimated using general data on the mean biomass of animals associated with each sieve, as described in Edgar (1990). Estimates of the daily productivity of benthic invertebrates were calculated using biomass estimates for each animal and the equation $\mathrm{P}=0.0049^{\star} \mathrm{B}^{0.80} \mathrm{~T}^{0.89}$, which relates daily macrobenthic productivity $\mathbf{P}\left(\mu \mathrm{g} \mathrm{d}^{-1}\right)$ to ash-free dry weight $\mathrm{B}(\mu \mathrm{g})$ and water temperature $\mathrm{T}\left({ }^{\circ} \mathrm{C}\right)$ (Edgar, 1990). Data collected in replicate samples from each site were amalgamated in the present study, with the mean density value for each species at each site used in analyses (Edgar et al., 1999).

Biological relationships between sites were assessed using non-metric multidimensional scaling (MDS), as run by the PRIMER program (Carr, 1996). The similarity matrices used in MDS were calculated between paired sites using the Bray-Curtis similarity coefficient after double root transformation, as recommended by Faith et al. (1987) and Clarke (1993). The usefulness of the MDS display of relationships between sites is indicated by the stress statistic, which if $<0 \cdot 1$ indicates that the depiction of relationships is good, and if $>0.2$ that the depiction is poor (Clarke, 1993).

The density of individual species at the different sites was related to human activity using Spearman rank correlation coefficients. Species with high correlation values were positively associated with human activity while species with low values were negatively associated. Human activity was quantified using two indices, the human population density and percent cleared land in catchment. In order to weight these two indices for activity in the immediate vicinity of the estuary, data for each site were calculated as the mean of the value for the total catchment area and the value for the estuarine drainage area. The estuarine drainage area was defined as that region draining directly into the estuary rather than into the major associated river. The latter, in turn, was defined as occurring upstream of the point on 1:100 000 topographic maps where river banks were shown as separate lines, where the $10 \mathrm{~m}$ contour line crossed the river flow line, or where probable obstructions to tidal flow such as gorges were shown on maps (see Edgar et al., 2000).

Data on human population density and percent cleared land in catchments were obtained by overlaying GIS maps of catchment areas on digital population census and land use data sets (see Edgar et al., 1999, 2000), following methods more fully described by Graddon (1997). Data on catchment land use were obtained by categorization of satellite images of the Tasmanian mainland, but were not available for estuaries on Bass Strait islands. Data on total annual runoff into estuaries were obtained by calibrating a GIS rainfall prediction model based on flow data from 63 gauged rivers and 504 rainfall stations (Edgar et al., 2000), while salinity, tidal range and the silt/clay content of sediments associated with each site were recorded in the field. Salinity was measured at $0.1 \mathrm{~m}$ depth below the surface during low tide, tidal range was estimated as the distance between the high tide layer of deposited flotsam and maximum retreat of water on the day of sampling, and the silt/clay $(<63 \mu \mathrm{m})$ particle fraction was determined for aggregated sediments collected in shallow subtidal $(0 \cdot 3-0 \cdot 7 \mathrm{~m})$ depths. The latter variable was assessed at only 39 of the 55 sites sampled for macrobenthos.

Spearman rank correlation coefficients $\left(R_{s}\right)$ relating animal abundance and human activity for all invertebrate species across the 55 sites were used to calculate an index of anthropogenic disturbance $(D I)$ for each site:

$$
D I_{n}=\mathrm{E} R_{s_{i}} \star n_{i} / N
$$

where $n_{i}$ is the abundance of species $i$ and $N$ is total abundance of all species at the site. This index was calculated so that estuarine assemblages at Tasmanian sites could be categorized with respect to human impact using a single univariate value. The index 
eliminated much of the largely irrelevant information associated with each site that was caused by variation in natural environmental factors such as salinity and tidal range rather than human impact, as well as ' noise ' contributed by species that occurred at a wide range of sites but were not greatly influenced by human activity. Such an index was considered a potentially useful tool when monitoring changes to impacted habitats over time, and for assessing the spatial scale of impacts.

Comparable disturbance indices were also calculated using data on the estimated productivity and biomass of macrofauna at each site $\left(D I_{p}=\mathrm{E} R_{s_{i}} \star p_{i} / P\right.$ and $D I_{b}=\mathrm{E} R_{s_{i}} \star b_{i} / B$, where $p_{i}$ and $b_{i}$ represent total productivity and biomass of species $i$, and $P$ and $B$ represent total productivity and biomass of all macrofauna at the site).

Assessment of $D I$ in the present study was partly confounded by circularity of calculations, in that indices were calculated using information on relationships between species abundance and disturbance to estuaries (population density), and then tested using aggregated site information against the same population density variable. Ideally, derivation and assessment data sets should have been independent because any variable will show a relatively high correlation with $D I$ when used also to calculate the weightings for each species that form the basis of $D I$. To assess the scale of this bias, weightings for species were calculated using a dummy variable composed to random numbers, and then the correlation coefficient between $D I$ and the original dummy variable calculated. The Abundance/Biomass Comparison (ABC) method of Warwick (1986) was also applied to the macrofaunal data set to assess whether this procedure has value in identifying sites subjected to human disturbance. In this procedure, the cumulative abundance and biomass of species ranked in order to importance in a sample were graphically compared for samples collected at four sites in estuaries with highest and lowest population densities in catchments. Only samples collected at or below low water mark were used in this analysis. Samples from higher tidal levels were excluded because they tended to be influenced by terrestrial as well as estuarine processes (Edgar \& Barrett, unpublished data). Patterns of variation in the fauna were highly consistent between 0.3 and $0.7 \mathrm{~m}$ depth at sites examined. The hypothesis tested using the ABC procedure was that anthropogenic impact causes elevated densities of small individuals, hence total abundance is dominated by relatively few species compared to biomass under disturbed conditions (Warwick et al., 1987).

\section{Results}

\section{Relationships between species abundance and human activity}

Macrofaunal relationships between sites are depicted using MDS in Figure 1. The plot shown is threedimensional because the equivalent two-dimensional plot produced a relatively poor description of the data (stress $=0 \cdot 12$ cf. $0 \cdot 17$ ). The bubble plot overlay of human population density in catchment indicates that faunal assemblages varied between sites in a pattern related to population density (Figure 1). This correspondence occurred almost exclusively along MDS axis 1 . The site with negligible population density at the extreme right of axis 1 was the Wanderer Estuary, an aberrant location with extremely low faunal productivity and little similarity to any other site (Edgar et al., 1999). Percentage cleared land in catchment and silt-clay content of sediments at the sample site showed relatively poor relationships with macrofaunal assemblages.

Relationships between densities of species at sites and the level of anthropogenic disturbance, as assessed using Spearman rank correlation coefficients that relate total animal abundance at each site with human population density and percent cleared land, are listed for abundant species in Table 1. Significance values have not been assigned to correlation coefficients because of uncertainty caused by the large number of species investigated during the study increasing the probability of Type I error, and the generally small number of sites at which each species was recorded affecting the probability of Type II error. A total of 157 of the 390 taxa collected were recorded at a single site only, with the great majority of these animals considered marine vagrants (Edgar et al., 1999).

Population density was generally more highly correlated with species abundance than percent cleared land, and therefore appeared to be the better discriminator of human impacts. Population density produced a highest correlation coefficient of 0.51 for Tellina deltoidalis and a minimum value of -0.45 for Limnoporeia kingi, compared to maximum and minimum values amongst all species of 0.37 and -0.29 using percent cleared land data.

The six widespread species most highly correlated with human population density (Table 1) were infaunal species typically associated with mudflats. Other species that were also highly correlated with population density but not shown in Table 1 because they occurred at less than 12 sites, and so have a high chance of being spuriously correlated, were also 
Human population density
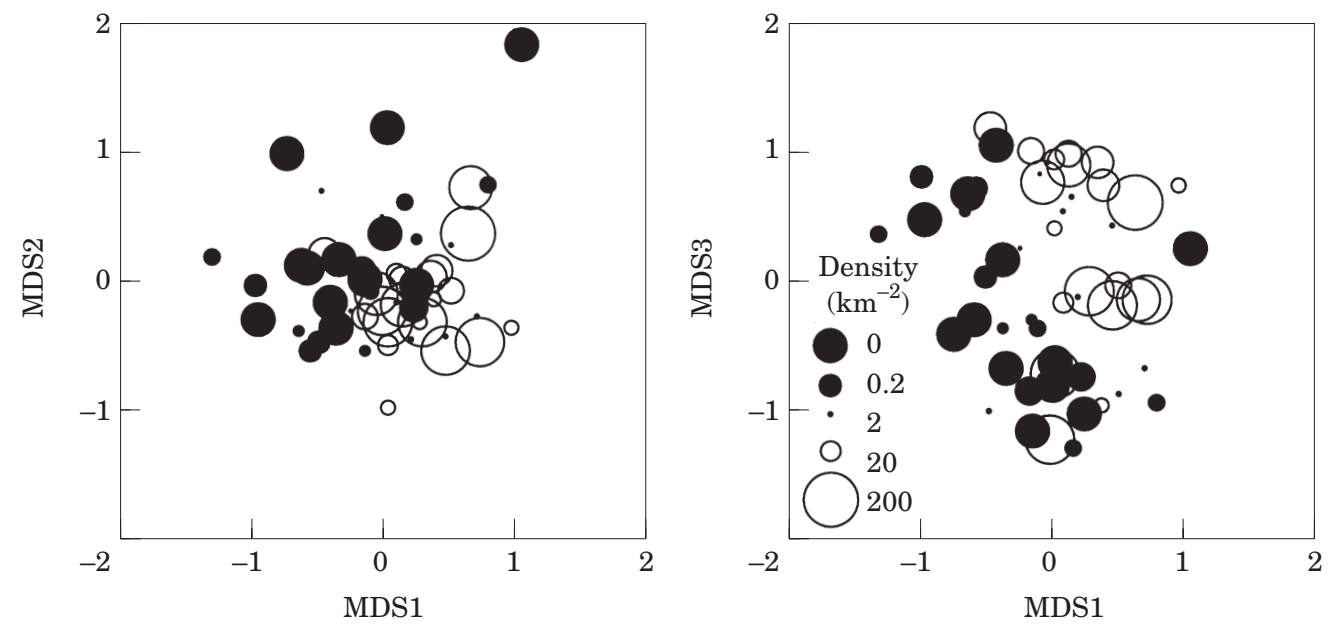

Cleared land
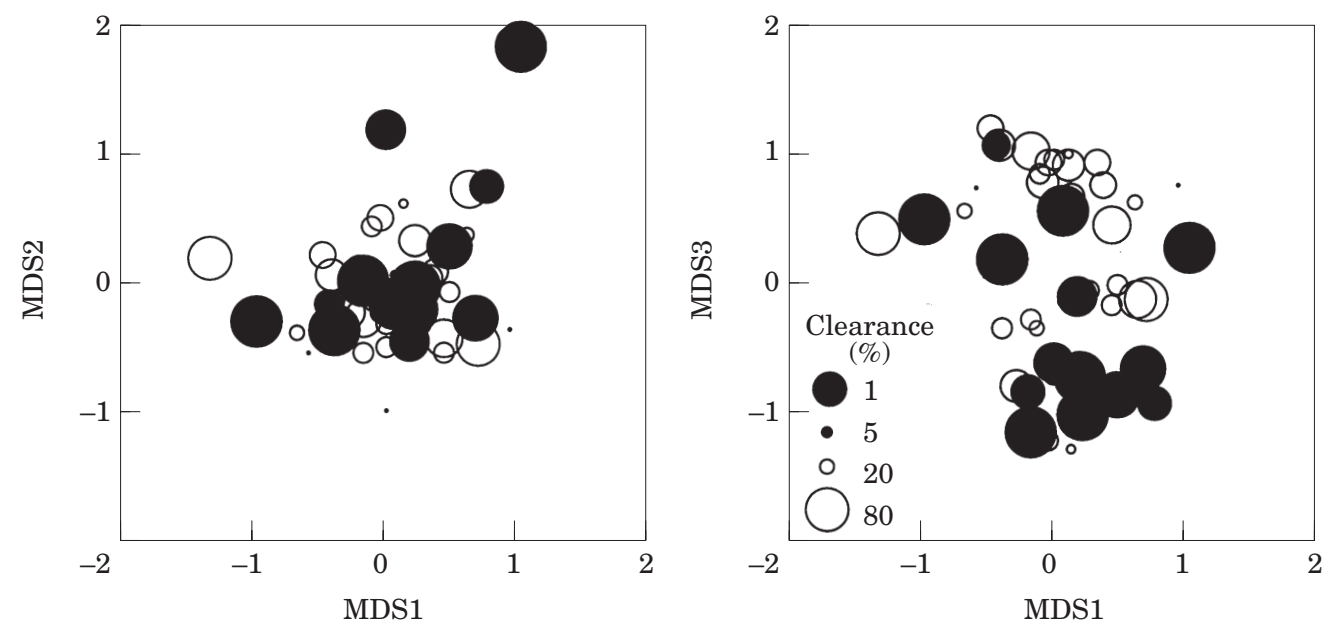

Silt/clay fraction
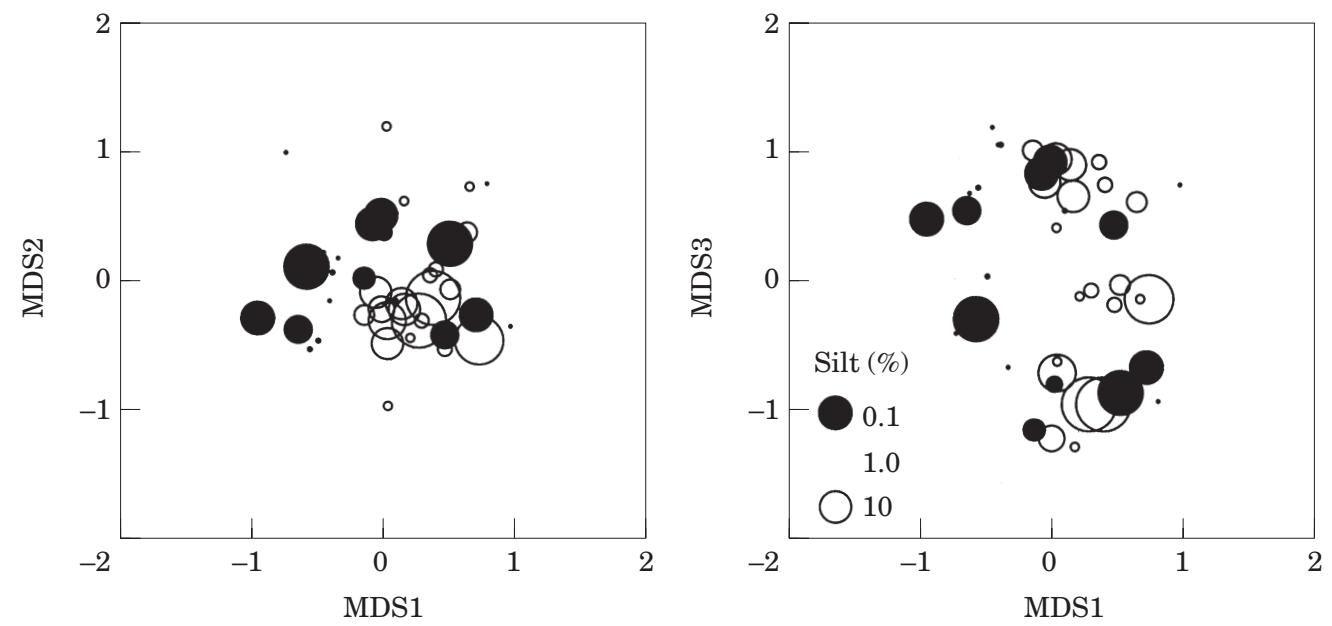

FIGURE 1. Bubble plot overlays of human population density in catchment, percentage cleared land in catchment and percentage of silt and clay $(<63 \mu \mathrm{m})$ in sediments on 3-d MDS depiction of faunal relationships between sites. Note that MDS axes values provide an arbitrary frame of reference. 
TABLE 1. Spearman rank correlation coefficients $\left(R_{s}\right)$ relating abundance at different sites of widespread species (i.e. the 46 species present at 12 or more sites) with population density (Pop) and $\%$ cleared land (Clear). Infaunal or epifaunal habit of species is also shown

\begin{tabular}{|c|c|c|c|c|}
\hline Species & Habit & Group & Pop $R_{s}$ & Clear $R_{s}$ \\
\hline Tellina deltoidalis & Infaunal & Bivalve & $0 \cdot 51$ & $0 \cdot 37$ \\
\hline Magelona sp. & Infaunal & Polychaete & $0 \cdot 39$ & $0 \cdot 30$ \\
\hline Lumbrineris sp. 1 & Infaunal & Polychaete & $0 \cdot 39$ & $0 \cdot 35$ \\
\hline Macrophthalmus latifrons & Infaunal & Crab & $0 \cdot 36$ & $0 \cdot 24$ \\
\hline Nephtys australiensis & Infaunal & Polychaete & $0 \cdot 32$ & $0 \cdot 15$ \\
\hline Callianassa arenosa & Infaunal & Shrimp & $0 \cdot 30$ & $0 \cdot 17$ \\
\hline Nassarius burchardi & Epifaunal & Gastropod & $0 \cdot 26$ & $0 \cdot 15$ \\
\hline Neanthes vaalii & Infaunal & Polychaete & $0 \cdot 24$ & $0 \cdot 26$ \\
\hline Paragrapsus gaimardii & Epifaunal & Crab & $0 \cdot 22$ & $0 \cdot 19$ \\
\hline Gammaropsis sp. 1 & Epifaunal & Amphipod & $0 \cdot 21$ & $0 \cdot 01$ \\
\hline Actaecia bipleura & Epifaunal & Isopod & $0 \cdot 19$ & $0 \cdot 27$ \\
\hline Phyllodoce sp. & Epifaunal & Polychaete & $0 \cdot 19$ & $0 \cdot 35$ \\
\hline Limnoporeia yarrague & Epifaunal & Amphipod & $0 \cdot 18$ & $0 \cdot 22$ \\
\hline Australonereis ehlersi & Infaunal & Polychaete & $0 \cdot 13$ & 0.02 \\
\hline Dimorphostylis colefaxi & Epifaunal & Cumacean & $0 \cdot 09$ & $-0 \cdot 04$ \\
\hline Heteromastus sp. & Infaunal & Polychaete & $0 \cdot 08$ & $0 \cdot 11$ \\
\hline Mictyris platycheles & Infaunal & Crab & 0.07 & $0 \cdot 14$ \\
\hline Exoediceroides?maculosus & Infaunal & Amphipod & $0 \cdot 07$ & 0 \\
\hline Nassarius pauperatus & Epifaunal & Gastropod & $0 \cdot 07$ & -0.08 \\
\hline Mysella donaciformis & Infaunal & Bivalve & $0 \cdot 07$ & $0 \cdot 10$ \\
\hline Paracorophium cf excavatum & Infaunal & Amphipod & $0 \cdot 06$ & $0 \cdot 20$ \\
\hline Macrobrachium sp. & Epifaunal & Shrimp & $0 \cdot 06$ & -0.09 \\
\hline Pseudolana concinna & Epifaunal & Isopod & -0.01 & $-0 \cdot 17$ \\
\hline Xenostrobus inconstans & Epifaunal & Bivalve & $-0 \cdot 04$ & $0 \cdot 02$ \\
\hline Paracalliope australis & Epifaunal & Amphipod & -0.05 & $0 \cdot 17$ \\
\hline Nemertean sp. 1 & Infaunal & Nemertean & -0.06 & -0.05 \\
\hline Melita $\mathrm{sp}$. & Epifaunal & Amphipod & -0.07 & $0 \cdot 20$ \\
\hline Arthritica semen & Infaunal & Bivalve & $-0 \cdot 11$ & $0 \cdot 01$ \\
\hline Euzonus sp. & Infaunal & Polychaete & $-0 \cdot 11$ & $0 \cdot 05$ \\
\hline Katelysia scalarina & Infaunal & Bivalve & $-0 \cdot 11$ & $0 \cdot 03$ \\
\hline Capitella sp. 2 & Infaunal & Polychaete & $-0 \cdot 12$ & 0 \\
\hline Tatea rufilabrus & Epifaunal & Gastropod & $-0 \cdot 12$ & $0 \cdot 02$ \\
\hline Leitoscoloplos normalis & Infaunal & Polychaete & $-0 \cdot 12$ & $-0 \cdot 15$ \\
\hline Boccardiella sp. & Infaunal & Polychaete & $-0 \cdot 13$ & 0.09 \\
\hline Salinator fragilis & Epifaunal & Gastropod & $-0 \cdot 14$ & $-0 \cdot 12$ \\
\hline Simplisetia aequisetis & Infaunal & Polychaete & $-0 \cdot 15$ & $-0 \cdot 25$ \\
\hline Perinereis vallata & Infaunal & Polychaete & $-0 \cdot 19$ & $0 \cdot 04$ \\
\hline Chironomid spp. & Epifaunal & Insect & $-0 \cdot 20$ & $0 \cdot 01$ \\
\hline Zeacumantus diemenensis & Epifaunal & Gastropod & $-0 \cdot 21$ & 0.09 \\
\hline ?Mysella $\mathrm{sp}$ & Infaunal & Bivalve & $-0 \cdot 24$ & $-0 \cdot 10$ \\
\hline Ascorhis victoriae & Epifaunal & Gastropod & $-0 \cdot 27$ & $-0 \cdot 23$ \\
\hline Exosphaeroma sp. & Epifaunal & Isopod & $-0 \cdot 28$ & $-0 \cdot 22$ \\
\hline Hydrococcus brazieri & Epifaunal & Gastropod & $-0 \cdot 28$ & 0 \\
\hline Amarinus lacustris & Epifaunal & Crab & -0.35 & $-0 \cdot 21$ \\
\hline Eubittium lawleyanum & Epifaunal & Gastropod & $-0 \cdot 38$ & $-0 \cdot 29$ \\
\hline Limnoporeia kingi & Epifaunal & Amphipod & $-0 \cdot 45$ & $-0 \cdot 17$ \\
\hline
\end{tabular}

mudflat dwelling species (e.g. the bivalve Notospisula trigonella $-R_{s}=0.40$ and the crabs Heloecius cordiformis $-R_{s}=0 \cdot 28$ and Helograpsus haswellianus$\left.R_{s}=0 \cdot 24\right)$. By contrast, the six widespread species showing strongest negative correlations with population density were epifaunal species. This included three species that generally associate with sandflats in similar salinity and tidal height conditions to the mudflat species (Eubittium lawleyanum, Hydrococcus brazieri and Exosphaeroma sp.). Most additional species with strong negative correlations with population density that were not included in Table 1 because of restricted distribution were also sandflat inhabitants (e.g. the bivalve Wallucina assimilis $-R_{s}=-0.32$ and 
TABLE 2. Disturbance index values calculated using abundance $\left(D I_{n}\right)$, biomass $\left(D I_{b}\right)$ and productivity $\left(D I_{p}\right)$ data. $D I_{n}$ was also calculated using a reduced 30 species data set and using percent cleared land rather than human population density as the initial correlate. DI indices have been rescaled for comparisons in the range from 0 to 10 (where 0 indicates the site examined during the study with lowest $D I$ and 10 the site with highest $D I$ ). Human population density and percent cleared land in catchment are also shown. Land clearance data were not available for seven estuaries on Bass Strait islands

\begin{tabular}{|c|c|c|c|c|c|c|c|c|}
\hline Estuary & Date & $D I_{n}$ & $D I_{b}$ & $D I_{p}$ & $\begin{array}{c}D I_{n} \\
\text { Reduced }\end{array}$ & $\begin{array}{c}D I_{n} \\
\% \text { Cleared }\end{array}$ & Population & $\%$ Cleared \\
\hline Sea Elephant & 24 Feb 97 & $1 \cdot 09$ & $5 \cdot 90$ & $0 \cdot 39$ & $2 \cdot 09$ & $9 \cdot 38$ & $0 \cdot 23$ & - \\
\hline Yellow Rock & 23 Feb 97 & $0 \cdot 85$ & 0 & $0 \cdot 78$ & $2 \cdot 12$ & $10 \cdot 00$ & $1 \cdot 20$ & - \\
\hline North East Inlet & 8 May 97 & $2 \cdot 41$ & $5 \cdot 34$ & $1 \cdot 34$ & $2 \cdot 64$ & $4 \cdot 50$ & $0 \cdot 12$ & - \\
\hline Patriarch Inlet & 9 May 97 & $2 \cdot 26$ & $5 \cdot 82$ & $1 \cdot 88$ & $1 \cdot 72$ & $4 \cdot 24$ & 0.06 & - \\
\hline Cameron Inlet & 9 May 97 & $2 \cdot 43$ & $2 \cdot 26$ & $1 \cdot 33$ & 1.95 & $4 \cdot 97$ & $0 \cdot 12$ & - \\
\hline Modder & 12 May 97 & 4.03 & $4 \cdot 22$ & $2 \cdot 64$ & $1 \cdot 77$ & $6 \cdot 69$ & 0 & - \\
\hline Rices & 6 May 97 & 0 & $3 \cdot 90$ & $0 \cdot 19$ & $1 \cdot 23$ & $6 \cdot 48$ & 0 & - \\
\hline Welcome Inlet & $21 \mathrm{Feb} 97$ & $0 \cdot 01$ & $2 \cdot 64$ & $2 \cdot 01$ & $2 \cdot 01$ & $4 \cdot 23$ & $0 \cdot 57$ & $22 \cdot 0$ \\
\hline Mosquito Inlet & 22 Feb 97 & $3 \cdot 11$ & $5 \cdot 78$ & $1 \cdot 23$ & $2 \cdot 52$ & $2 \cdot 86$ & 0 & $1 \cdot 43$ \\
\hline East Inlet & 15 Jan 97 & $2 \cdot 78$ & $7 \cdot 80$ & $2 \cdot 23$ & $2 \cdot 55$ & $2 \cdot 69$ & $7 \cdot 61$ & $49 \cdot 2$ \\
\hline Black/Dip & 14 Jan 97 & $7 \cdot 44$ & $7 \cdot 42$ & $5 \cdot 71$ & $7 \cdot 06$ & $3 \cdot 26$ & $1 \cdot 78$ & $33 \cdot 2$ \\
\hline Detention & 13 Jan 97 & $5 \cdot 69$ & $6 \cdot 88$ & $4 \cdot 54$ & $9 \cdot 15$ & $2 \cdot 26$ & $3 \cdot 86$ & $33 \cdot 3$ \\
\hline Cam & 26 Sep 96 & $5 \cdot 89$ & $8 \cdot 28$ & $5 \cdot 36$ & $8 \cdot 08$ & $5 \cdot 49$ & $74 \cdot 4$ & $53 \cdot 8$ \\
\hline Blythe & 16 Jan 97 & $6 \cdot 02$ & $9 \cdot 21$ & $7 \cdot 63$ & $8 \cdot 99$ & $4 \cdot 72$ & $25 \cdot 7$ & $28 \cdot 2$ \\
\hline Leven & 25 Sep 96 & $7 \cdot 14$ & $5 \cdot 62$ & $3 \cdot 36$ & $9 \cdot 95$ & $1 \cdot 24$ & $71 \cdot 6$ & $45 \cdot 6$ \\
\hline Leven & 17 May 97 & $7 \cdot 37$ & $3 \cdot 91$ & $3 \cdot 84$ & $9 \cdot 39$ & $1 \cdot 44$ & $71 \cdot 6$ & $45 \cdot 6$ \\
\hline Don & 24 Sep 96 & $5 \cdot 84$ & $10 \cdot 00$ & $7 \cdot 46$ & $8 \cdot 73$ & $4 \cdot 79$ & $152 \cdot 0$ & $69 \cdot 8$ \\
\hline Port Sorell & 17 Jan 97 & $6 \cdot 23$ & 0.69 & $3 \cdot 14$ & $8 \cdot 77$ & $1 \cdot 97$ & $11 \cdot 9$ & $31 \cdot 2$ \\
\hline Tamar/Low Head & 16 Dec 96 & $6 \cdot 14$ & $5 \cdot 55$ & $4 \cdot 83$ & $8 \cdot 17$ & $3 \cdot 05$ & $39 \cdot 8$ & $36 \cdot 00$ \\
\hline Tamar/Paper Beach & 17 Dec 96 & $9 \cdot 15$ & $7 \cdot 08$ & $10 \cdot 00$ & $9 \cdot 91$ & 0 & $39 \cdot 8$ & $36 \cdot 00$ \\
\hline Tamar/Paper Beach & 17 Jun 97 & $10 \cdot 00$ & $5 \cdot 70$ & $9 \cdot 86$ & $10 \cdot 00$ & $0 \cdot 13$ & $39 \cdot 8$ & $36 \cdot 00$ \\
\hline Pipers & 20 Nov 96 & $5 \cdot 53$ & $7 \cdot 00$ & $4 \cdot 55$ & $7 \cdot 67$ & $2 \cdot 85$ & $3 \cdot 21$ & $28 \cdot 7$ \\
\hline Tomahawk & 22 Nov 96 & $4 \cdot 30$ & $7 \cdot 14$ & $3 \cdot 09$ & $7 \cdot 40$ & $5 \cdot 25$ & 0.63 & $52 \cdot 5$ \\
\hline Boobyalla & 19 Nov 96 & $4 \cdot 81$ & $7 \cdot 29$ & $5 \cdot 14$ & $2 \cdot 27$ & $5 \cdot 59$ & $1 \cdot 16$ & $41 \cdot 1$ \\
\hline Little Musselroe Bay & 14 Nov 96 & $1 \cdot 26$ & $4 \cdot 05$ & $0 \cdot 33$ & $1 \cdot 12$ & $3 \cdot 12$ & $0 \cdot 39$ & $72 \cdot 7$ \\
\hline Ansons Bay & 12 Nov 96 & $4 \cdot 93$ & $6 \cdot 52$ & $3 \cdot 28$ & $4 \cdot 34$ & $4 \cdot 40$ & $2 \cdot 82$ & $8 \cdot 21$ \\
\hline Big Lagoon & 13 Nov 96 & $2 \cdot 46$ & $5 \cdot 95$ & $1 \cdot 64$ & $2 \cdot 06$ & $8 \cdot 86$ & $0 \cdot 14$ & $1 \cdot 28$ \\
\hline Georges Bay & 11 Nov 96 & $6 \cdot 40$ & $3 \cdot 92$ & $6 \cdot 83$ & $9 \cdot 64$ & $3 \cdot 17$ & $17 \cdot 7$ & $15 \cdot 6$ \\
\hline Hendersons Lagoon & 13 Nov 96 & $3 \cdot 14$ & $6 \cdot 01$ & $4 \cdot 87$ & $6 \cdot 81$ & $6 \cdot 06$ & 1.04 & $25 \cdot 7$ \\
\hline Bryans Lagoon & 13 May 97 & $1 \cdot 36$ & $2 \cdot 04$ & $0 \cdot 79$ & $2 \cdot 10$ & $9 \cdot 48$ & 0 & 0 \\
\hline Great Swanport & 23 Dec 96 & $2 \cdot 62$ & $5 \cdot 44$ & $2 \cdot 24$ & $2 \cdot 87$ & $7 \cdot 39$ & $0 \cdot 43$ & $22 \cdot 8$ \\
\hline Lisdillon & 17 Sep 96 & $2 \cdot 68$ & 0 & $4 \cdot 14$ & $2 \cdot 41$ & $7 \cdot 31$ & $3 \cdot 66$ & $42 \cdot 9$ \\
\hline Prosser & 16 Sep 96 & $5 \cdot 17$ & $6 \cdot 41$ & $6 \cdot 13$ & $5 \cdot 65$ & $6 \cdot 06$ & $10 \cdot 3$ & $17 \cdot 8$ \\
\hline Earlham Lagoon & 20 Aug 96 & $1 \cdot 06$ & $2 \cdot 37$ & $2 \cdot 90$ & $1 \cdot 05$ & $6 \cdot 13$ & $0 \cdot 42$ & $28 \cdot 2$ \\
\hline Pittwater & 23 Oct 96 & $3 \cdot 57$ & $2 \cdot 93$ & $4 \cdot 17$ & $4 \cdot 42$ & $2 \cdot 78$ & $26 \cdot 5$ & $55 \cdot 8$ \\
\hline Derwent/Cornelian Bay & 24 Apr 96 & $6 \cdot 97$ & $9 \cdot 06$ & $7 \cdot 23$ & $8 \cdot 35$ & $5 \cdot 22$ & $164 \cdot 0$ & $30 \cdot 3$ \\
\hline Derwent/Cornelian Bay & 13 Feb 97 & $7 \cdot 20$ & $8 \cdot 64$ & $7 \cdot 38$ & $8 \cdot 33$ & $4 \cdot 18$ & $164 \cdot 0$ & $30 \cdot 3$ \\
\hline Derwent/Bridgewater & 21 Oct 96 & $7 \cdot 18$ & $3 \cdot 87$ & $6 \cdot 22$ & $2 \cdot 17$ & $6 \cdot 31$ & $164 \cdot 0$ & $30 \cdot 3$ \\
\hline Derwent/Claremont & 21 Oct 96 & $5 \cdot 58$ & $6 \cdot 21$ & $4 \cdot 63$ & $5 \cdot 45$ & $6 \cdot 46$ & $164 \cdot 0$ & $30 \cdot 3$ \\
\hline Browns & 2 Jul 96 & $6 \cdot 16$ & $9 \cdot 04$ & $7 \cdot 90$ & $9 \cdot 32$ & $2 \cdot 98$ & $279 \cdot 0$ & $21 \cdot 0$ \\
\hline Huon/Eggs and Bacon & 6 Nov 96 & $7 \cdot 33$ & $5 \cdot 14$ & $3 \cdot 69$ & $8 \cdot 39$ & $4 \cdot 90$ & $7 \cdot 92$ & $12 \cdot 2$ \\
\hline Huon/Brabazon & 5 Nov 96 & $6 \cdot 37$ & $5 \cdot 46$ & $5 \cdot 35$ & $9 \cdot 04$ & $3 \cdot 55$ & $7 \cdot 92$ & $12 \cdot 2$ \\
\hline Huon/Cradoc & 31 Oct 96 & $5 \cdot 74$ & $5 \cdot 76$ & $4 \cdot 68$ & $7 \cdot 79$ & $6 \cdot 82$ & $7 \cdot 92$ & $12 \cdot 2$ \\
\hline Huon/Cradoc & 16 May 97 & $5 \cdot 82$ & $7 \cdot 03$ & $5 \cdot 27$ & $5 \cdot 76$ & $6 \cdot 75$ & $7 \cdot 92$ & $12 \cdot 2$ \\
\hline Hastings & 9 Jan 97 & $5 \cdot 66$ & $6 \cdot 61$ & $4 \cdot 78$ & $9 \cdot 02$ & $5 \cdot 64$ & 0.95 & 0.57 \\
\hline Southport Lagoon & 19 Jun 97 & 0.05 & $2 \cdot 82$ & $0 \cdot 15$ & $1 \cdot 58$ & $5 \cdot 06$ & 0 & 0 \\
\hline Cloudy Bay/southwest & 18 May 97 & $1 \cdot 22$ & $2 \cdot 07$ & $1 \cdot 85$ & $2 \cdot 52$ & $6 \cdot 17$ & $0 \cdot 32$ & $10 \cdot 7$ \\
\hline Cloudy Bay/northeast & 24 May 97 & $0 \cdot 84$ & $4 \cdot 68$ & $1 \cdot 92$ & $2 \cdot 36$ & $5 \cdot 22$ & $0 \cdot 32$ & $10 \cdot 7$ \\
\hline Cockle Creek & 7 Jan 97 & $7 \cdot 40$ & $6 \cdot 60$ & $3 \cdot 39$ & $7 \cdot 53$ & $2 \cdot 38$ & $1 \cdot 73$ & 0 \\
\hline New River Lagoon & 18 Feb 97 & $2 \cdot 42$ & $0 \cdot 51$ & 0.93 & $2 \cdot 08$ & $8 \cdot 88$ & 0 & 0 \\
\hline Bathurst Harbour & 19 Feb 97 & $3 \cdot 48$ & $8 \cdot 61$ & $2 \cdot 72$ & $7 \cdot 33$ & $8 \cdot 21$ & 0.03 & 0 \\
\hline Payne Bay & 19 Feb 97 & $0 \cdot 72$ & $1 \cdot 93$ & $1 \cdot 86$ & $2 \cdot 06$ & $5 \cdot 24$ & 0 & 0 \\
\hline Wanderer & $20 \mathrm{Feb} 97$ & $6 \cdot 70$ & $8 \cdot 49$ & $5 \cdot 77$ & $5 \cdot 26$ & $1 \cdot 66$ & 0 & 0 \\
\hline
\end{tabular}


TABle 2. (Continued)

\begin{tabular}{|c|c|c|c|c|c|c|c|c|}
\hline Estuary & Date & $D I_{n}$ & $D I_{b}$ & $D I_{p}$ & $\begin{array}{c}D I_{n} \\
\text { Reduced }\end{array}$ & $\begin{array}{c}D I_{n} \\
\% \text { Cleared }\end{array}$ & Population & $\%$ Cleared \\
\hline Macquarie/heads & 30 Sep 96 & $5 \cdot 38$ & $7 \cdot 30$ & $4 \cdot 50$ & $5 \cdot 26$ & $4 \cdot 78$ & $2 \cdot 94$ & $0 \cdot 31$ \\
\hline Macquarie/Swan Basin & 23 Jun 97 & $3 \cdot 66$ & $2 \cdot 07$ & $1 \cdot 58$ & $1 \cdot 74$ & $6 \cdot 81$ & $2 \cdot 94$ & $0 \cdot 31$ \\
\hline Henty & 25 Jun 97 & $1 \cdot 07$ & 0.03 & 0 & $5 \cdot 26$ & $9 \cdot 10$ & 0.07 & $0 \cdot 19$ \\
\hline Pieman & 24 Jun 97 & $4 \cdot 50$ & $3 \cdot 28$ & $2 \cdot 75$ & 0 & $5 \cdot 85$ & 0.53 & $0 \cdot 96$ \\
\hline Nelson Bay & 14 Jan 97 & $3 \cdot 44$ & $2 \cdot 03$ & $1 \cdot 65$ & $0 \cdot 71$ & $6 \cdot 15$ & 0 & $0 \cdot 64$ \\
\hline Arthur & 13 Jan 97 & $3 \cdot 81$ & $2 \cdot 39$ & $2 \cdot 54$ & 1.98 & $7 \cdot 51$ & $0 \cdot 31$ & $17 \cdot 3$ \\
\hline
\end{tabular}

the gastropods Diala suturalis $-R_{s}=-0.25$ and Haminoea maugensis $\left.-R_{s}=-0 \cdot 24\right)$.

Calculation of the disturbance index $D I_{n}$ for each site using Spearman rank correlation coefficients and abundance data indicated a wide range of values between sites. The site with the highest DI, and therefore the one most dominated by species typical of disturbed (i.e. heavily populated) conditions, was Paper Beach (Tamar Estuary) while Rices River was found to be the least disturbed. $D I_{n}$ values for all sites sampled are listed in Table 2 .

$D I_{n}$ appears useful as an environmental indicator of anthropogenic disturbance of sites. It retains a high correlation with population density $\left(R_{s}=0.74\right)$ and, to a lesser extent, with percent cleared land $\left(R_{s}=0.55\right)$, but was not strongly correlated with the major physical variable salinity $\left(R_{s}=-0 \cdot 31\right)$. In addition, bubble plots overlaying $D I_{n}$ values on $M D S$ results showed a very good separation of sites (Figure 2), particularly for the plot of MDS axis 1 vs axis 3. $D I_{n}$ therefore has a high degree of faunal consistency and discriminates well between sites.

\section{Disturbance indices based on reduced species data}

In order to determine whether calculations of $D I_{n}$ were dominated by a limited number of influential species, this index was recalculated using data from the 15 species most highly positively and negatively correlated with human population density, as listed in Table 3.

Correlation of $D I$ values with human population density for sites calculated using the reduced species data set was similar to that for the full data set $\left(R_{s}=0.73\right.$ cf. 0.74$)$, hence little information had been lost. The bubble plots of $D I$ overlaying MDS results were also similar to plots obtained using all species (Figure 2).

\section{Disturbance indices based on biomass and productivity data}

$D I$ was also calculated using data on the total biomass $\left(D I_{b}\right)$ and estimated productivity $\left(D I_{p}\right)$ of species at each site rather than total numbers $\left(D I_{n}\right)$. The bubble plot overlays on MDS results (Figure 3) indicated that sites separated less strongly on the basis of $D I_{b}$ than on $D I_{p}$ or $D I_{n}$. Relatively few sites possessed high $D I_{p}$ values (Table 2).

$D I_{n}$ and $D I_{p}$ both showed very little change over time, with $D I_{p}$ marginally more stable (see Table 2, where $D I$ values for sites sampled on two occasions are shown). By contrast, $D I_{b}$ fluctuated considerably between sampling occasions, depending partly on the occasional collection of very large individuals. After DI was rescaled to range between 0 and 10 , the average standard deviation at sites between times was 0.84 for $D I_{b}$ compared with 0.25 and 0.24 for $D I_{n}$ and $D I_{p}$ respectively.

$D I_{p}$ was the best performing of all indices examined in terms of retaining its correlation with human population density $\left(R_{s}=0 \cdot 78\right.$, Table 4$)$, while the correlation of $D I_{b}$ was relatively poor $\left(R_{s}=0 \cdot 40\right) . D I_{p}$ also maintained a better correlation with the other disturbance variable, percent cleared land, than $D I_{n}$ and was less influenced by the major physical variables salinity, tidal range and total annual runoff (Table 4$). D I_{p}$ maintained a high correlation with the silt/clay fraction of sediments, tidal range and total annual runoff. This, given the high correlation of population density itself with these variables, was expected. $D I_{n}, D I_{b}$ and $D I_{p}$ were all moderately negatively correlated $\left(-0.49<R_{s}<-0.28\right)$ with the density of animals at sites but were not greatly affected by the total biomass, total estimated productivity or total number of species.

Assessment of DI using species weightings calculated for a dummy variable composed of random 
Full data set
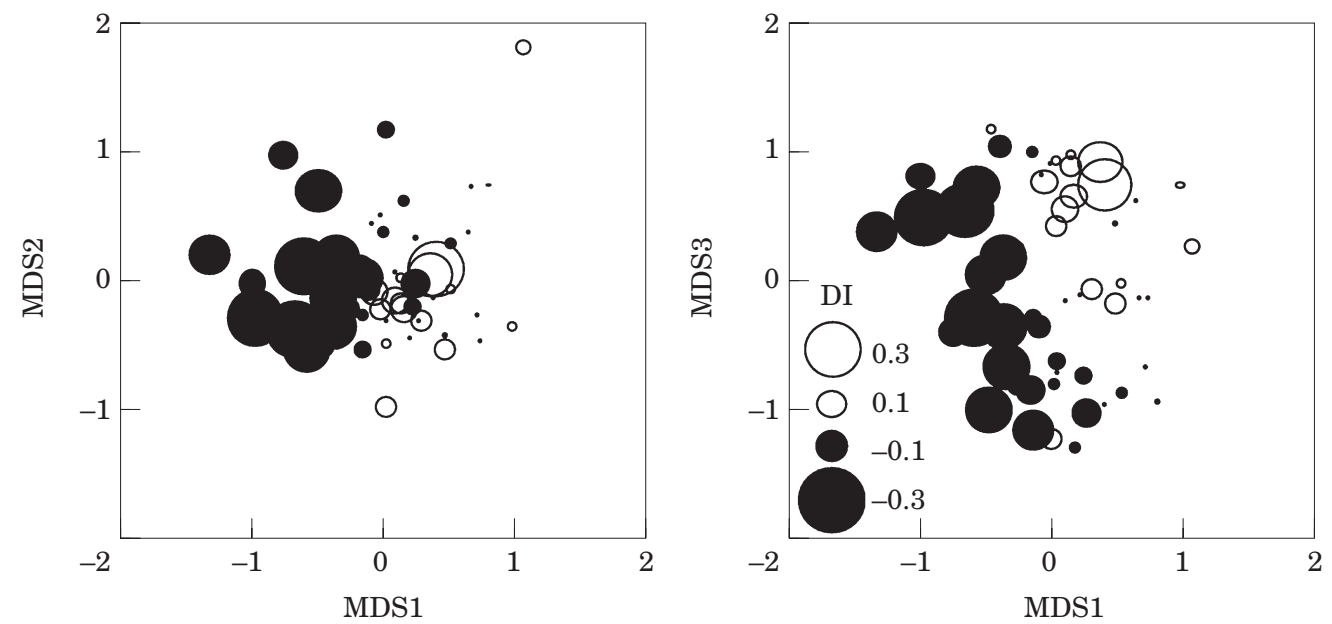

Reduced data set
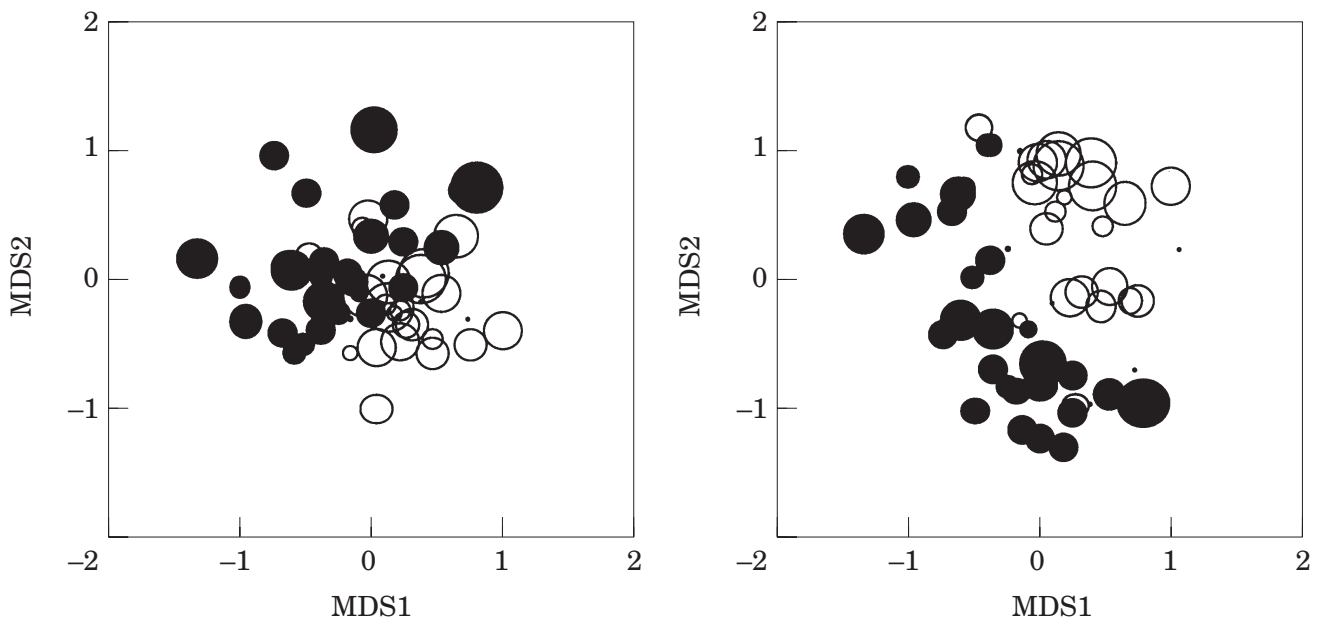

FIGURE 2. Bubble plot overlays of $D I_{n}$ values on 3-D MDS depiction of faunal relationships between sites. Analyses based on full faunal data set and reduced 30 species data set are shown.

numbers, and then back correlated against $D I$, produced a mean correlation coefficient of 0.49 for 50 dummy variables. This value was slightly higher than the correlation coefficient relating percent cleared land in catchment with the $D I_{n}$ values calculated using that variable $(=0 \cdot 44$, Table 4$)$ and the correlation coefficient for $D I_{b}$ and population density $(=0 \cdot 40$, Table 4). $D I$ calculated using percent cleared land and $D I_{b}$ therefore both possessed negligible relationship with the biota.

By contrast, comparable correlation coefficients based on human population density data were very high for both $D I_{n}\left(R_{s}=0.74\right)$ and $D I_{p}\left(R_{s}=0 \cdot 78\right)$. In terms of the proportion of total variance explained $\left(R^{2}\right)$, statistical bias assessed using dummy variables explained $24 \% \quad\left(=0.49^{2}\right)$, leaving $31 \% \quad\left(=0.74^{2}-\right.$ $\left.0 \cdot 49^{2}\right)$ and $37 \%\left(=0 \cdot 78^{2}-0 \cdot 49^{2}\right)$ of the remaining
$76 \%$ attributable to population density for $D I_{n}$ and $D I_{p}$, respectively. After correction for circularity in calculations, the correlation coefficients between DI and human population density therefore remained high at $0.64(=\sqrt{ }(0.31 / 0.76))$ and $0.70 \quad(=\sqrt{ }(0.37 /$ 0.76)) for $D I_{n}$ and $D I_{p}$, respectively.

\section{Effects of estuary type}

Because $D I$ values and human population density were both highly correlated with other environmental variables, the possibility that the strong relationship between $D I$ and population density was driven primarily by covariation with environmental factors rather than a direct relationship was examined by partitioning estuaries on the basis of geomorphological and hydrological type. Tasmanian estuaries were 
TABLE 3. Spearman rank correlation coefficients $\left(R_{s}\right)$ relating human population density in catchments with total abundance of animals for 15 species showing highest and lowest correlations

\begin{tabular}{llll}
\hline Species & $r_{s}$ & \multicolumn{1}{c}{ Species } & $r_{s}$ \\
\hline Tellina deltoidalis & $0 \cdot 51$ & Limnoporeia kingi & $-0 \cdot 45$ \\
Notospisula trigonella & $0 \cdot 40$ & Eubittium lawleyanum & $-0 \cdot 38$ \\
Magelona sp. & $0 \cdot 39$ & Amarinus lacustris & $-0 \cdot 35$ \\
Lumbrineris sp. 1 & $0 \cdot 39$ & Wallucina assimilis & $-0 \cdot 32$ \\
Macrophthalmus latifrons & $0 \cdot 36$ & Scolecolepides sp. & $-0 \cdot 31$ \\
Nephtys australiensis & $0 \cdot 32$ & Hydrococcus brazieri & $-0 \cdot 28$ \\
Callianassa arenosa & $0 \cdot 30$ & Exosphaeroma sp. & $-0 \cdot 28$ \\
Barantolla lepte & $0 \cdot 30$ & Olganereis edmonsi & $-0 \cdot 27$ \\
Heloecius cordiformis & $0 \cdot 28$ & Ascorhis victoriae & $-0 \cdot 27$ \\
Corophium sp. & $0 \cdot 27$ & Glycerid sp. 1 & $-0 \cdot 25$ \\
Nassarius burchardi & $0 \cdot 26$ & Diala suturalis & $-0 \cdot 25$ \\
Placamen placida & $0 \cdot 25$ & Solemya sp. & $-0 \cdot 24$ \\
Glycerid sp. 2 & $0 \cdot 25$ & ?Mysella sp. & $-0 \cdot 24$ \\
Helograpsus haswellianus & $0 \cdot 24$ & Haminoea maugensis & $-0 \cdot 24$ \\
Neanthes vaalii & $0 \cdot 24$ & Cyamiomacra mactroides & $-0 \cdot 22$ \\
\hline
\end{tabular}

subdivided in an associated study (Edgar et al., 2000) into nine groups on the basis of biologically-important environmental characteristics (viz., presence of a seaward barrier, tidal range, salinity, estuary size and river runoff), with six of these estuary groups investigated for macrofauna at more than three sites. Relationships between $D I$ and human population density within these six estuary categories (barred low-salinity estuary, open polyhaline estuary, marine inlet, mesotidal river estuary, microtidal drowned river valley and open microtidal river estuary) were investigated using Analysis of Covariance (ANCOVA). Results based on $D I$ as dependent variable, population density as independent variable (log $\mathrm{x}+0 \cdot 1$ ), and estuary type as grouping variable are presented in Table 5 .

None of the three $D I$ indices showed a significant interaction between estuary type and log population density (analysis with interaction in Table 5), hence the slopes of the regression equations in different estuary types were similar and the interaction variable was removed from the second analysis. $D I_{n}$ and $D I_{p}$ both showed extremely strong relationships with population density after the effects of estuary were taken into account (analysis without interaction in Table 5, $P<0.001)$; however, $D I_{b}$ possessed no significant relationship with population density but a significant relationship with estuary type. The overall significant relationship between $D I_{b}$ and population density resulted from different estuary types possessing differing mean levels of $D I_{b}$, and population density also differing significantly between the estuary types.
When comparable ANCOVAs were conducted using four other important environmental variables (tidal range, log total annual runoff, salinity and $\log$ silt/clay content of sediments) in place of human population density, no significant $(\alpha=0.05)$ interactions were detected, hence slopes were also considered homogenous in these ANCOVAs. Salinity and $\log$ total annual runoff were found to vary significantly with $D I$ once the effects of different estuary types were taken into account (Table 6), although significant variation in $D I$ occurred between estuaries of different types. $D I_{n}$ varied significantly with tidal range within different estuary groups, while $D I_{n}$ and $D I_{p}$ also varied with the percentage silt/clay content of sediments.

Relationships between $D I_{n}$, human population density in catchment, silt/clay content of sediments and estuary groups are shown as scatterplots in Figure 4. These relationships were non-linear, with negligible change in both $D I$ and the silt/clay content of sediments occurring for sites with population densities between 0 and $1 \mathrm{~km}^{-2}$, rapid increases in dependent variables occurring when densities were between 1 and $10 \mathrm{~km}^{-2}$, and then little difference between sites in catchments with densities $>10 \mathrm{~km}^{-2}$.

\section{Abundance/biomass comparisons}

Results of Warwick's (1986) ABC procedure for sites in estuaries with highest human population densities and sites lacking population within catchments are shown in Figure 5. Biomass curves lacked any consistent pattern in relation to abundance curves, rather than showing greater elevation at undisturbed sites, as 
Biomass
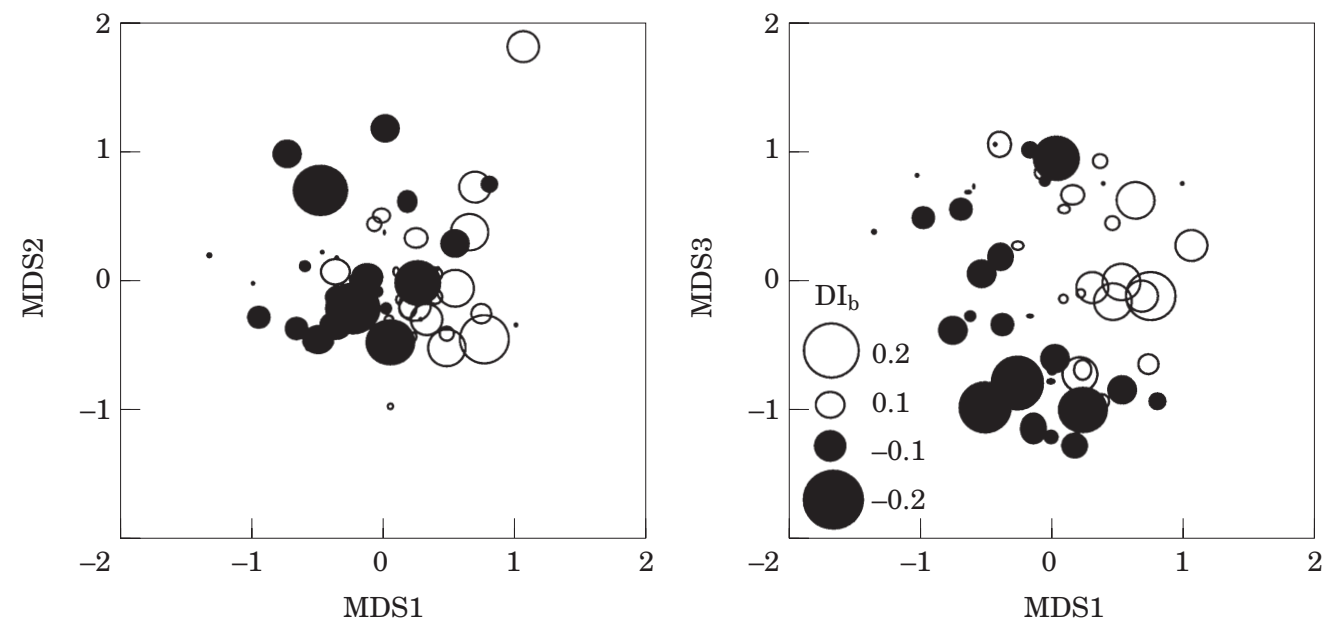

Productivity
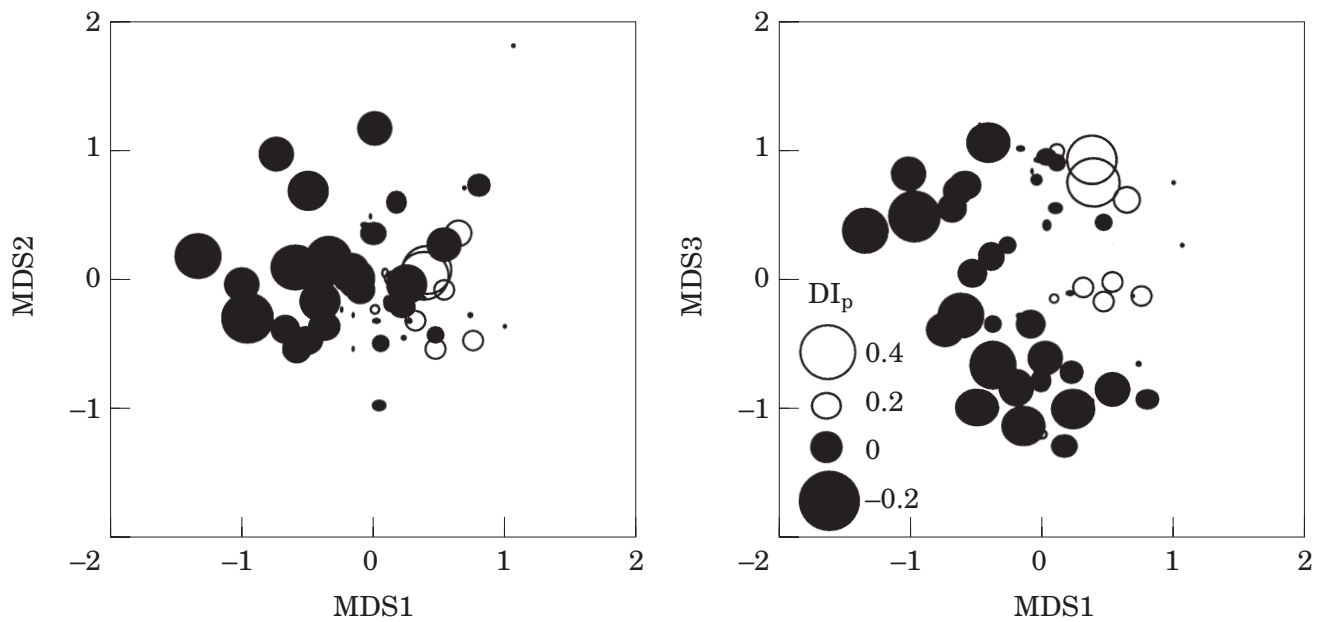

FIGURE 3. Bubble plot overlays of $D I_{b}$ values calculated using biomass data and $D I_{p}$ values calculated using estimated productivity data on MDS results.

would be expected if anthropogenic impact caused high densities of small species. The only noticeable trend in the complete data set, which includes sites not shown in Figure 5, related to the salinity of sites. Marine sites (e.g. Kelly Basin) were often dominated in terms of biomass by a few relatively large bivalves, while freshwater-influenced sites were generally inhabited by dense populations of small amphipods and hydrobiid gastropods.

\section{Discussion}

\section{Biological indicators of human disturbance}

The two anthropogenic disturbance indices proposed in this study, $D I_{n}$ and $D I_{p}$, appear to be sensitive bio- logical indicators of human impacts within Tasmanian estuaries. Both indices were highly correlated with human population density within the catchment area, discriminated between sites with different faunal characteristics (Figure 2), and showed negligible change between different seasons. While these indices should prove useful for monitoring biological changes in Tasmanian estuaries over time, they have restricted geographic applicability because of their basis on correlation coefficients for individual species.

The index based on faunal abundance, $D I_{n}$, has a single major advantage over the index based on faunal productivity, $D I_{n}$, in that it is relatively simple to measure and comprehend. The advantage of $D I_{p}$ over $D I_{n}$ is that it is more biologically meaningful because $D I_{p}$ is not heavily biased by either large- or small-sized 
TABLE 4. Spearman rank correlation coefficients relating disturbance indices calculated using abundance $\left(D I_{n}\right)$, biomass $\left(D I_{b}\right)$ and productivity $\left(D I_{p}\right)$ data, human population density and environmental variables. In addition to the full data set, $D I_{n}$ was calculated using a reduced 30 species data set and using percent cleared land rather than human population density as the initial correlate

\begin{tabular}{|c|c|c|c|c|c|c|}
\hline Variable & $D I_{n}$ & $D I_{b}$ & $D I_{p}$ & $\begin{array}{c}D I_{n} \\
\text { Reduced }\end{array}$ & $\begin{array}{c}D I_{n} \\
\text { \% Cleared }\end{array}$ & Population \\
\hline Population density & $0 \cdot 74$ & $0 \cdot 40$ & $0 \cdot 78$ & $0 \cdot 73$ & $0 \cdot 41$ & $1 \cdot 00$ \\
\hline$\%$ Cleared land & $0 \cdot 37$ & $0 \cdot 28$ & $0 \cdot 46$ & $0 \cdot 43$ & $0 \cdot 44$ & $0 \cdot 67$ \\
\hline Salinity & $-0 \cdot 30$ & $-0 \cdot 13$ & $-0 \cdot 26$ & $-0 \cdot 11$ & $0 \cdot 31$ & $-0 \cdot 21$ \\
\hline Tidal range & $0 \cdot 50$ & $0 \cdot 38$ & $0 \cdot 47$ & 0.59 & $0 \cdot 61$ & 0.57 \\
\hline Latitude & $-0 \cdot 18$ & $-0 \cdot 02$ & $-0 \cdot 24$ & $-0 \cdot 20$ & $0 \cdot 17$ & $-0 \cdot 16$ \\
\hline Total annual runoff & 0.52 & $0 \cdot 12$ & $0 \cdot 47$ & $0 \cdot 35$ & $0 \cdot 10$ & $0 \cdot 49$ \\
\hline$\%$ Silt/clay & 0.59 & $0 \cdot 29$ & $0 \cdot 60$ & $0 \cdot 45$ & $0 \cdot 18$ & 0.65 \\
\hline Species number & $-0 \cdot 10$ & $-0 \cdot 15$ & -0.02 & $0 \cdot 13$ & $0 \cdot 36$ & $0 \cdot 11$ \\
\hline Faunal density & -0.49 & $-0 \cdot 28$ & $-0 \cdot 38$ & -0.55 & -0.53 & $-0 \cdot 26$ \\
\hline Faunal biomass & -0.04 & -0.09 & -0.05 & $0 \cdot 12$ & $0 \cdot 40$ & $0 \cdot 14$ \\
\hline Faunal productivity & -0.08 & $-0 \cdot 15$ & $-0 \cdot 11$ & $0 \cdot 04$ & $0 \cdot 28$ & $0 \cdot 12$ \\
\hline
\end{tabular}

TABLE 5. Results of Analyses of Covariance investigating the relationship between DI calculated using abundance, biomass and productivity data and log-transformed human population density in catchments associated with estuaries grouped into six geomorphological and hydrological types

\begin{tabular}{|c|c|c|c|c|c|c|c|c|c|c|}
\hline \multirow[b]{2}{*}{ Effect } & \multirow[b]{2}{*}{$\mathrm{DF}$} & \multicolumn{3}{|c|}{ Abundance $\left(D I_{n}\right)$} & \multicolumn{3}{|c|}{ Biomass $\left(D I_{b}\right)$} & \multicolumn{3}{|c|}{ Productivity $\left.D I_{p}\right)$} \\
\hline & & MS & $F$ & $P$ & MS & $F$ & $P$ & MS & $F$ & $P$ \\
\hline \multicolumn{11}{|l|}{ Analysis with interaction } \\
\hline Estuary & 5 & $8 \cdot 854$ & $5 \cdot 026$ & $0 \cdot 001$ & $11 \cdot 293$ & $2 \cdot 782$ & $0 \cdot 030$ & $2 \cdot 553$ & $1 \cdot 619$ & $0 \cdot 177$ \\
\hline Population & 1 & $14 \cdot 149$ & $8 \cdot 032$ & $0 \cdot 007$ & $4 \cdot 747$ & $1 \cdot 169$ & $0 \cdot 286$ & $26 \cdot 291$ & $16 \cdot 668$ & $<0.001$ \\
\hline Population $\times$ estuary & 5 & $4 \cdot 005$ & $2 \cdot 273$ & $0 \cdot 065$ & $4 \cdot 872$ & $1 \cdot 200$ & $0 \cdot 326$ & $1 \cdot 056$ & $0 \cdot 669$ & 0.649 \\
\hline Error & 41 & $1 \cdot 762$ & & & $4 \cdot 059$ & & & $1 \cdot 577$ & & \\
\hline \multicolumn{11}{|c|}{ Analysis without interaction } \\
\hline Estuary & 5 & $5 \cdot 803$ & $2 \cdot 894$ & $0 \cdot 024$ & $19 \cdot 259$ & $4 \cdot 644$ & $0 \cdot 002$ & $3 \cdot 400$ & $2 \cdot 236$ & $0 \cdot 067$ \\
\hline Population & 1 & $53 \cdot 615$ & $26 \cdot 735$ & $<0.001$ & $3 \cdot 508$ & $0 \cdot 846$ & $0 \cdot 363$ & $71 \cdot 345$ & $46 \cdot 919$ & $<0.001$ \\
\hline Error & 46 & $2 \cdot 005$ & & & $4 \cdot 147$ & & & $1 \cdot 521$ & & \\
\hline
\end{tabular}

species (Edgar, 1990; Edgar \& Shaw, 1995; Warwick \& Clarke, 1993). Also, the productivity of a species is approximately proportional to total food consumption, total respiration and total reproductive output of that species, and so provides a relative index of its trophic importance (Edgar, 1993). By contrast, indices which relate the abundance of a species to others have much less biological meaning because they can be influenced by one or two species of small size that contribute relatively little to the functioning of the community. Small species tend to be far more abundant in samples than large species.

An additional advantage of $D I_{p}$ over $D I_{n}$ was that it showed a higher correlation with percent cleared land, and so possibly responded to a wider range of human impacts (Table 4). DI $I_{p}$ was also less strongly correlated with natural environmental variables (salinity, tidal range, total annual runoff) and the total animal density of samples $\left(r_{s}=-0.38 \mathrm{cf}\right.$. -0.49$)$.

The extent to which $D I_{n}$ and $D I_{p}$ are affected by natural physical factors and total sample size needs to be clarified in future studies. This is best done by manipulation of the level of anthropogenic impact independently of changes in physical factors. Such studies should also examine whether the biological indicators are affected by a large or narrow range of anthropogenic impacts, including siltation, nutrification, reduced oxygen concentrations and heavy metal concentrations.

Widespread human impacts in estuaries are likely to prove difficult to detect using methods other than disturbance indices, given that human impacts are often less obvious than pronounced relationships with natural environmental variables such as salinity (see 
TABLE 6. Results of ANCOVAs relating DI calculated using abundance, biomass and productivity data to environmental variables (tidal range, log total annual runoff, salinity and $\log \%$ silt/clay content of sediments) for estuaries grouped into six geomorphological and hydrological types

\begin{tabular}{|c|c|c|c|c|c|c|c|c|c|c|}
\hline \multirow[b]{2}{*}{ Effect } & \multirow[b]{2}{*}{$\mathrm{DF}$} & \multicolumn{3}{|c|}{ Abundance $\left(D I_{n}\right)$} & \multicolumn{3}{|c|}{ Biomass $\left(D I_{b}\right)$} & \multicolumn{3}{|c|}{ Productivity $\left(D I_{p}\right)$} \\
\hline & & MS & $F$ & $P$ & MS & $F$ & $P$ & MS & $F$ & $P$ \\
\hline \multicolumn{11}{|l|}{ Tidal range } \\
\hline Estuary type & 5 & $21 \cdot 19$ & $7 \cdot 629$ & $<0 \cdot 001$ & 23 & $5 \cdot 447$ & $0 \cdot 001$ & $17 \cdot 16$ & $5 \cdot 585$ & $<0.001$ \\
\hline Tide & 1 & $18 \cdot 09$ & $6 \cdot 512$ & $0 \cdot 014$ & $0 \cdot 014$ & 0.003 & $0 \cdot 954$ & 0 & 0 & 0.99 \\
\hline Error & 46 & $2 \cdot 778$ & & & $4 \cdot 224$ & & & $3 \cdot 072$ & & \\
\hline \multicolumn{11}{|l|}{ Total annual runoff } \\
\hline Estuary type & 5 & $13 \cdot 55$ & $4 \cdot 342$ & $0 \cdot 003$ & $34 \cdot 05$ & $8 \cdot 541$ & $<0.001$ & $15 \cdot 06$ & $5 \cdot 032$ & 0.001 \\
\hline Total annual runoff & 1 & $2 \cdot 322$ & $0 \cdot 744$ & $0 \cdot 393$ & $10 \cdot 95$ & $2 \cdot 747$ & $0 \cdot 104$ & $3 \cdot 646$ & $1 \cdot 218$ & $0 \cdot 275$ \\
\hline Error & 46 & $3 \cdot 121$ & & & $3 \cdot 986$ & & & $2 \cdot 992$ & & \\
\hline \multicolumn{11}{|l|}{ Salinity } \\
\hline Estuary type & 5 & $21 \cdot 34$ & $6 \cdot 796$ & $<0 \cdot 001$ & $32 \cdot 72$ & $7 \cdot 761$ & $<0.001$ & $19 \cdot 75$ & $6 \cdot 46$ & $<0.001$ \\
\hline Salinity & 1 & $1 \cdot 438$ & $0 \cdot 458$ & $0 \cdot 502$ & $0 \cdot 362$ & 0.086 & $0 \cdot 771$ & 0.661 & $0 \cdot 216$ & 0.644 \\
\hline Error & 46 & $3 \cdot 14$ & & & $4 \cdot 216$ & & & $3 \cdot 057$ & & \\
\hline \multicolumn{11}{|l|}{$\%$ silt/clay } \\
\hline Estuary type & 5 & $17 \cdot 88$ & $11 \cdot 03$ & $<0 \cdot 001$ & 15 & $3 \cdot 453$ & $0 \cdot 014$ & $14 \cdot 21$ & $8 \cdot 274$ & $<0.001$ \\
\hline Silt/clay & 1 & $7 \cdot 552$ & $4 \cdot 66$ & 0.039 & $3 \cdot 28$ & 0.755 & $0 \cdot 392$ & $12 \cdot 79$ & $7 \cdot 451$ & 0.011 \\
\hline Error & 30 & $1 \cdot 621$ & & & $4 \cdot 344$ & & & $1 \cdot 717$ & & \\
\hline
\end{tabular}

Edgar et al., 2000). Species richness provided a poor indication of human disturbance in Tasmanian estuaries, largely because mudflats and sandflats contained faunas of similar species richness, as evident in the low correlation between population density and species number (Table 4). The Abundance/Biomass Comparison method for detecting human impacts (Warwick, 1986) also lacked usefulness because many of the infaunal mudflat species associated with high human population densities possessed relatively large body size and biomass (e.g. Tellina deltoidalis). Dauer et al. (1993) have described similar problems in using the $\mathrm{ABC}$ method to detect stressed estuarine communities.

Furthermore, multivariate methods that incorporate environmental variables into a predictive model based on undisturbed habitats, and then assess the difference between observed and predicted assemblages (e.g. Moss et al., 1987), are also unsuitable for identifying impacted sites if sediment characteristics are included in the model. Such models will always predict a mudflat fauna for site with muddy sediments when sediment characteristics are incorporated as a habitat factor into the model.

\section{Anthropogenic impacts on biota in Tasmanian estuaries}

Macrofaunal species living in the upper and middle reaches of Tasmanian estuaries are adapted to a physical environment that undergoes rapid and ex- treme fluctuations in salinity, temperature, water flow and turbidity. Most estuarine species also appear to be resilient to the effects of human disturbance. None of the major community metrics examined (species richness, faunal density, faunal biomass, faunal productivity) were found to be highly correlated with human population density or percent cleared land in catchments (Table 4).

Nevertheless, variation between estuaries in the disturbance indices $D I_{n}$ and $D I_{p}$ revealed clear differences between faunal assemblages in estuaries with different levels of human population density. Increasing population density was associated with increasing silt/clay content of sediments and with a reduction in number of epifaunal compared to infaunal species. These changes occurred consistently at relatively low human population densities, with virtually no overlap in $D I$ or silt/clay content of sediments between estuaries with $<1$ inhabitants $\mathrm{km}^{-2}$ and estuaries with $>10$ inhabitants $\mathrm{km}^{-2}$.

Tasmania is unusual in a global sense in retaining a large number of estuaries with $<1$ inhabitants $\mathrm{km}^{-2}$. If similar relationships with population density also apply in other regions, then biological communities in most temperature estuaries worldwide should be considered to have already undergone major shifts as a result of human activity. Comparable studies conducted in most countries would possibly show little anthropogenic effect because of a lack of 'pristine' reference sites. 

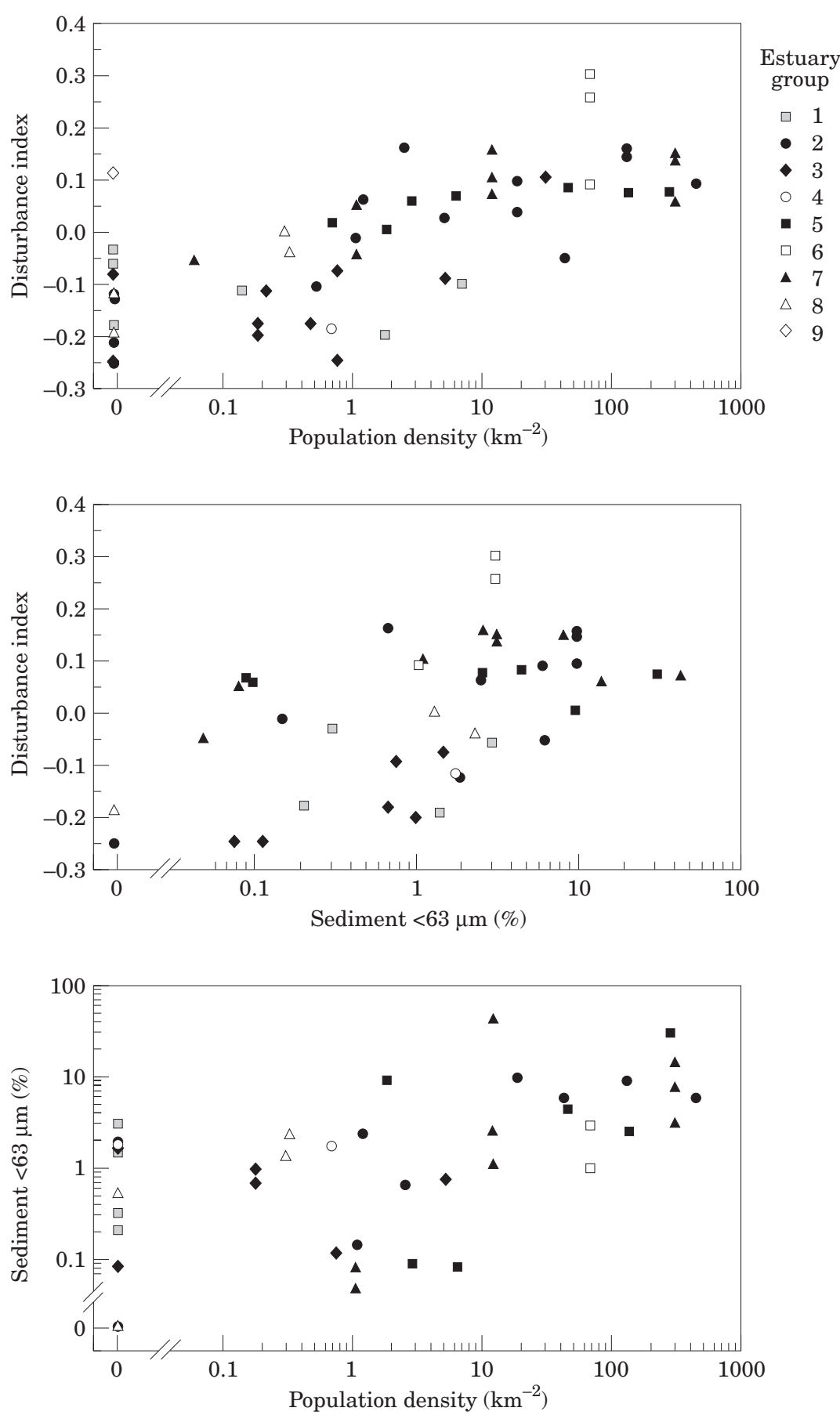

FIGURE 4. Scatterplots showing relationships between the faunal disturbance index $D I_{n}$ for sites located in estuaries grouped by morphological and hydrological similarities, human population density in catchments, and the silt/clay content of sediments. Note log format of density and silt/clay axes, and discontinuity of origin.

The correlations between human population density, silt/clay fraction of sediments and faunal composition may be due to direct dependence between these factors, or to indirect associations arising from shared relationships with other factors. Given the large number of studies that identify direct causal relation- ships between human activities in catchments and increased sediment loads (e.g. Campbell \& Doeg, 1989), the most likely hypothesis relating these factors is that anthropogenic activity in catchments and around estuary margins causes large inputs of fine suspended sediments that are transported to and 

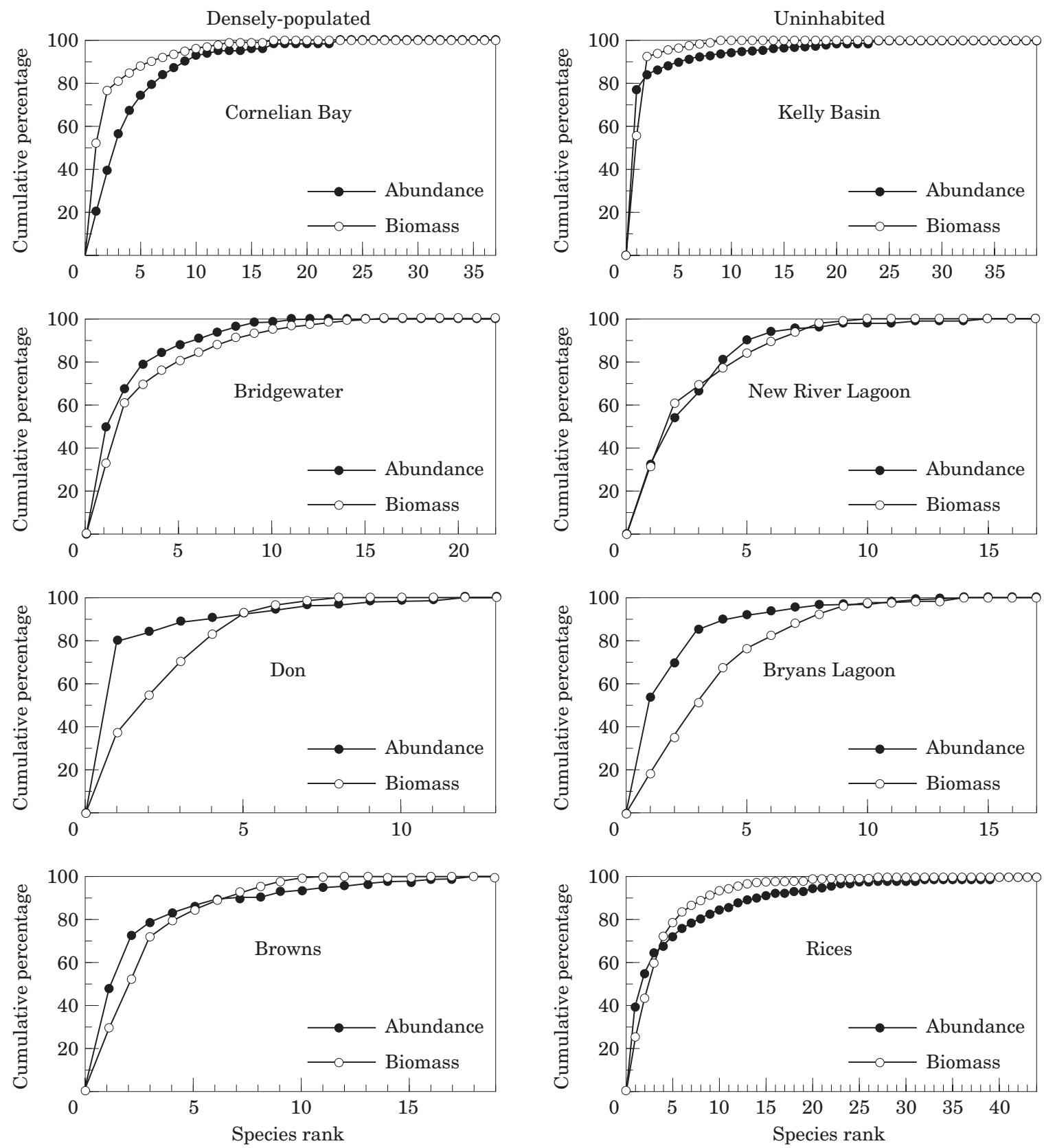

FIGURE 5. Abundance/biomass comparison plots for four sites in estuaries with highest human population densities and four sites in uninhabited estuaries.

deposited in the lower reaches of estuaries. These fine deposits coat the estuarine bed and eventually convert sandflats into mudflats, which attract infaunal species (such as Tellina deltoidalis and Magelona sp.) and displace epifauna (such as Eubittium lawleyanum and Zeacumantus diemenensis).

An alternative hypothesis is that human settlement, with resulting high population densities, preferentially occurs in estuaries with muddy rather than sandy sediments, perhaps because of richer soils for agricul- ture in the hinterland. While this hypothesis cannot be discounted outright, it is less likely than the previous hypothesis given the interspersion of estuaries with high and low human population densities studied around the state, and the extremely consistent relationships between human population density, silt-clay content of sediments and $D I_{n}$.

More importantly, results of the ANCOVAs indicated that $D I_{n}$ and $D I_{p}$ both increase with increasing human population density and silt/clay content of 
sediments within estuaries of similar geomorphological and hydrological type. Anecdotal information also consistently indicates that as human population density increases within catchments the foreshores of associated estuaries change from sandflat to mudflat. For example, Cornelian Bay in the lower Derwent estuary possessed a sandy beach until $c .1940$, but the shore has since degraded and now includes large quantities of silt and clay (Sustainable Development Advisory Council, 1996).

While siltation is clearly an important factor associated with human impacts within estuaries, other factors also probably contributed to faunal differences between estuaries with low and high human population densities. This is indicated by the substantially lower correlation coefficient between $D I$ and silt/clay content compared with $D I$ and population density (Table 4), and the poor correspondence between silt/clay content and faunal assemblage type (Figure 1). Amongst the most probable contributing factors associated with human impacts are nutrification, eutrophication, seagrass loss and discharge of urban and industrial waste. Manipulative experimental work is required to distinguish between the influences of these and other potentially-important factors.

\section{Acknowledgements}

Amongst the many people who made the project possible, we would particularly like to thank Dave Graddon for undertaking the GIS work. The project was supported by funding from Ocean Rescue 2000 (Environment Australia) and an ARC Research Fellowship.

\section{References}

Bamber, R. N. \& Spencer, J. F. 1984 The benthos of a coastal power station thermal discharge canal. Fournal of the Marine Biological Association of the United Kingdom 64, 603-623.

Boynton, W. R., Hagy, J. D., Murray, L., Stokes, C. \& Kemp, C. 1996 A comparative analysis of eutrophication patterns in a temperature coastal lagoon. Estuaries 19, 408-421.

Campbell, I. C. \& Doeg, T. J. 1989 Impact of timber harvesting and production on streams: a review. Australian fournal of Marine and Freshwater Research 40, 519-539.

Carlton, J. T. 1989 Man's role in changing the face of the ocean: biological invasions and implications for conservation of nearshore environment. Conservation Biology 3, 265-273.

Carr, M. R. 1996 PRIMER User Manual. Plymouth Routines in Multivariate Ecological Research. Plymouth Marine Laboratory, Plymouth, UK.

Clarke, K. R. 1993 Non-parametric multivariate analyses of changes in community structure. Australian fournal of Ecology 18, 117-143.

Cloern, J. E. 1996 Phytoplankton bloom dynamics in coastal ecosystems: a review with some general lessons from sustained investigation of San Francisco Bay, California. Reviews of Geophysics 34, 191-202.
Dauer, D. M., Luckenbach, M. W. \& Rodi, A. J. 1993 Abundance biomass comparison (ABC method): effects of an estuarine gradient, anoxic/hypoxic events and contaminated sediments. Marine Biology 116, 507-518.

De Jonge, V. N., Boynton, W. D., D’Elia, C. F., Elmgren, R. \& Welsh, R. L. 1994 Responses to developments in eutrophication in four different North Atlantic estuarine systems. In Changes in Fluxes in Estuaries: Implications from Science to Management (Dyer, K. R. \& Orth, R. J., eds). Olsen \& Olsen, Fredensborg, Denmark. pp. 179-196.

DeFur, P. L. \& Rader, D. N. 1995 Aquaculture in estuaries: Feast or famine? Estuaries 18, 2-9.

Edgar, G. J. 1990 The use of the size-structure of benthic macrofaunal communities to estimate faunal biomass and secondary production. Fournal of Experimental Marine Biology and Ecology 137, 195-214.

Edgar, G. J. 1993 Measurement of the carrying capacity of benthic habitats using a metabolic-rate based index. Oecologia 95, 115121.

Edgar, G. J. \& Shaw, C. 1995 The production and trophic ecology of shallow-water fish assemblages in southern Australia. III. General relationships between sediments, seagrasses, invertebrates and fishes. Fournal of Experimental Marine Biology and Ecology 194, 107-131.

Edgar, G. J., Barrett, N. S. \& Last, P. R. 1999 The distribution of macroinvertebrates and fishes in Tasmanian estuaries. Fournal of Biogeography 26, 1169-1190.

Edgar, G. J., Barrett, N. S., Graddon, D. J. \& Last, P. R. 2000 The conservation significance of estuaries: a classification of Tasmanian estuaries using ecological, physical and demographic attributes as a case study. Biological Conservation 92, 383-397.

Faith, D. P., Minchin, P. R. \& Belbin, L. 1987 Compositional dissimilarity as a robust measure of ecological distance. Vegetatio 69, 57-68.

Graddon, D. J. 1997 Characteristics of Tasmanian estuaries and catchments: physical attributes, population, and land use. Master of Environmental Studies thesis, University of Tasmania, Hobart.

Lavery, P. S., Lukatelich, R. J. \& McComb, A. J. 1991 Changes in the biomass and species composition of macroalgae in a eutrophic estuary. Estuarine and Coastal Shelf Science 33, 1-22.

McClelland, J. W., Valiela, I. \& Michener, R. H. 1997 Nitrogenstable isotope signatures in estuarine food webs: A record of increasing urbanization in coastal wetlands. Limnology and Oceanography 42, 930-937.

Moss, D., Furse, M. T., Wright, J. F. \& Armitrage, P. D. 1987 The prediction of the macro-invertebrate fauna of unpolluted running-water sites in Great Britain using environmental data. Freshwater Biology 17, 41-52.

Newcombe, C. P. \& Jensen, J. O. T. 1996 Channel suspended sediment and fisheries: A synthesis for quantitative assessment of risk and impact. North American fournal of Fisheries Management 16, 693-727.

Nixon, S. W. 1995 Coastal marine eutrophication: a definition, social causes, and future consequences. Ophelia 41, 199-219.

Ritz, D. A., Lewis, M. E. \& Shen, M. 1989 Response to organic enrichment of infaunal macrobenthic communities under salmonid seacages. Marine Biology 103, 211-214.

Rosenberg, D. M., Bodaly, R. A. \& Usher, P. J. 1995 Environmental and social impacts of large scale hydro-electric development: who is listening? Global Environmental Change 5, 127-148.

Saiz-Salinas, J. I., Ruiz, J. M. \& Frances-Zubillaga, G. 1996 Heavy metal levels in intertidal sediments and biota from the Bidasoa Estuary. Marine Pollution Bulletin 32, 69-71.

Schlacher, T. A. \& Wooldridge, T. H. 1996 Ecological responses to reductions in freshwater supply and quality in South Africa's estuaries: lessons for management and conservation. fournal of Coastal Conservation 2, 115-130.

Silberstein, K., Chiffings, A. W. \& McComb, A. J. 1986 The loss of seagrass in Cockburn Sound, Western Australia. III. The effect of epiphytes on the productivity of Posidonia australis Hook. $\mathrm{f}$. Aquatic Botany 24, 355-371. 


\section{G. J. Edgar and N. S. Barrett}

Sustainable Development Advisory Council 1996 State of the Environment, Tasmania, Volume 1-Conditions and Trends. Department of Environment \& Land Management, Hobart.

Valiela, I., Kremer, J., Lajtha, K., Geist, M., Seely, B., Brawley, J. \& Sham, C. H. 1997 Nitrogen loading from coastal watersheds to receiving estuaries: New method and application. Ecological Applications 7, 358-380.

van Dolah, R. F., Calder, D. R. \& Knott, D. M. 1984 Effects of dredging and open water disposal on benthic macroinvertebrates in a South Carolina estuary. Estuaries 7, 28-37.

Warwick, R. M. 1986 A new method for detecting pollution effects on marine macrobenthic communities. Marine Biology 92, 557-562.
Warwick, R. M., Pearson, T. H. \& Ruswahyuni 1987 Detection of pollution effects on marine macrobenthos: further evaluation of the species abundance/biomass method. Marine Biology 95, 193-200.

Warwick, R. M. \& Clarke, K. R. 1993 Comparing the severity of disturbance: a meta-analysis of marine macrobenthos community data. Marine Ecology Progress Series 92, 221-231.

Whitfield, A. K. 1986 Fish community structure response to major habitat changes within the littoral zone of an estuarine coastal lake. Environmental Biology of Fishes 17, 41-51.

Winemiller, K. O. \& Morales, N. E. 1989 Communidades de peces del Parque Nacional Corcovado luego del cese de las actividades mineras. Brenesia 31, 75-91. 